CORTES CABALLERO CARLOS - MP. PATOLOGO, JEFE LABORATORIO H.U.R.G.V?

ARENAS BUENAHORA ISAIAS - MD. GINE. COLOGO OBSTETRA EN EL H.U.R.G.V.

CABALLERO RUEDA ARNULFO - LABORATORIO CLINICO CLINICA SANTA TERESA.

\title{
Perfil Lipidico
}

\section{en Líquido Amniótico \\ de}

\section{Embarazadas a término}

$$
\text { - I - }
$$

\section{INTRODUCCION}

Es bien conocida la importancia de la determinación, de la relación lecitina y esfingomielina en jugo gástrico de recién nacidos y de las proteinas, en sangre del cordón umbilical, como pruebas para el diagnóstico del sindrome de distross respiratorio (1) poco se ha comentado sobre el valor de otras fracciones lipídicas, en el líquido amniótico, en el momento del trabajo de parto, como un índice de alteraciones en el metabolismo lipídico de la madre o el feto. (2-3) posteriormente. Esto y nuestro interés en los lipidos nos llevó a evaluar fosfolipidos, colestrol, y trigli- céridos antes del periódo expulsivo en cien (100) maternas que utilizaron los servicios del Hospital González Valencia de Bucaramanga.

Los resultados de esta experiencia se discuten en este trabajo.

$$
\text { - II - }
$$

\section{MATERIAL Y METODOS}

Se tomarón 100 mujeres de las que acudieron al servicio de Obstetricia y Ginecología del Hospital Universitario Ramón González Valencia, sin tener en cuenta, ni su edad, procedencia o raza; solo se descartó la presencia de enfermedad.

La punción se hizo por vía vaginal durante el trabajo de parto 
con feto vivo y cuando la dilatación cervical estaba entre 4 y $5 \mathrm{Cm}$.

Fueron descartados los líquidos meconiados o sanguinolentos; se utilizó aguja $\mathrm{N}^{0} 21$ con jeringa estéril de 10 c. c.

La muestra fue procesada en el Laboratorio de la Clínica Santa Teresa, sin previa centrifugación y todas las determinaciones hechaミ por la misma persona con control de calidad, teniendo en cuenta el conjunto de sueros y vacunas con concentración promedio ya conocida y acepíada en técnicas de Lakoratorio. (4).

Las determinaciones se realizaron según las técnicas enumeradas a continuación: para el colesterol se utilizó el método de anhíärido ascético ácido sulfurico (5) donde el colesterol forma junto con el anhídrido ascético y el ácido sulfúrico un compleịo coloreado que se mide luego fotométricamente; los fosfolípidos! se determinaron por el método de Connetry y col modificado (6) que precipita los fosfolipidos y las proteinas con eliminación del fósforo inorgánico, mineralización del complejo proteinas-fosfolipidos y determinación del fósforo por transformación y reducción para evaluar colorimetricamente al tenor equivalente de $4 \%$ de peso de fósforo en los fosfolipidos totales; los triglicéridos se determinaron según el método Zilversmit-van Handel modificado (7) donde el glycerol lik:erado por saponificación de los glicéridos del líquido amniótico es oxidado para formar formaldehido el cual reacciona con acetylacetona-amonia cuya concentración es determinada por adaptación colorimétrica le la reacción de hantzch descrita por Nash; finalmente para la determinación de los lípidos totales se utilizó la técnica de M. Merckotest lípidos totales: (8) según N. Zollner y K Kirsch en la cual después de calentar el líquido con ácido fosfórico-vainillina, reacción ésta en la que los lípidos producen un color rosado que se determina iotométricamente.

En Bucaramanga, se han hecho trabajos sobre determinación de lípidos totales y colesterol en líquido amniótico (3) pero no se han determinado previamente valores de fosfolípidos ni triglicéridos, por lo cual no consideramos los valores encontrados representativos para establecer normalidades; las cifras dadas por Frank E. Hytten y Tom Liind (9) en mujeres con $30-40$ semanas de emtarazo son en $\mathrm{mg} /$-: triglicéridos 13.6 - 2.6; colesterol libre $13.7+1$; fosfolípedos $45+$ 8.3 y lípidos totales 138.2 .

\section{COMENTARIOS}

Se agrupan cien (100) mujeres por edades comprendidas entre 15 a 19 años, 20 a 24,25 a 29 , 30 a 34 y 35 años o más observándose con el aumento de edad un ligero incremento en tri'glicéridos, colesterol y lípidos totales pero no en los fosfolípidos que mantienen un discreto descenso en su concentración.

Al observar el peso promedio de los recien nacidos encontramos en relación con la edad de la embarazada un ascenso proporcional 


\section{PROMEDIO DE VALORES OBTENIDOS}

\begin{tabular}{|c|c|c|c|c|c|c|c|}
\hline Edad & Colesterol & $\begin{array}{l}\text { Fosfolí- } \\
\text { pidos }\end{array}$ & $\begin{array}{l}\text { Triglicé- } \\
\text { ridos Lip. }\end{array}$ & Totales & $\begin{array}{c}\text { Peso } \\
\text { Recién } \\
\text { nacido }\end{array}$ & eso niños & P. P. Niñas \\
\hline $15-19$ & 23.05 & 63.34 & 88.50 & 443.18 & 3033 & 3308 & 2969.2 \\
\hline $20-24$ & 24.75 & 64.98 & 104.04 & 297.41 & 3275.6 & 3315 & 3238 \\
\hline $25-29$ & 22.43 & 53.62 & 87.92 & 361.45 & 3465.6 & 34.94 .4 & 3428.6 \\
\hline $30-34$ & 43.28 & 62.10 & 106.82 & 383.06 & 3720 & 3775 & 3683.3 \\
\hline-35 & 26.91 & 60.42 & 122.72 & 432.28 & 3485.7 & 3485.7 & 3433.3 \\
\hline Promedios: & 26.07 & 61.85 & 98.62 & 346.24 & 3396 & (grs) & \\
\hline
\end{tabular}

Los promedios de pesos estan en grs.

Los promedios de los componentes en mgrs/L. 
de los lípidos totales, triglicéridos y colesterol.

También se abserva como ya es de pleno conocimiento, un mayor peso en los niños que en las niñas. Encontramos que los niños cuyo peso está sobre 3.700 grs. mantienen un promedio de lípidos totales más elevados del promedio total hallado. En estos niños (17 en total) el promedio de peso fué de 4014.70 grs. mientras que el peso promedio total fue de 3396 grs; el promedio del valor de lípidos totales en el líquildo de estas embarazadas fué de $346.24 \mathrm{mg} / \mathrm{L}$. No se observó en cambio una variación significativa en esta relación con respecto a los fosfolípidos.

Los valores promedio de cada uno de los componentes analizados en relación a la edad podemos observarlo en la tabla adjunta, que comparadas con los valores dados por Hytten y Lind (9) difieren en todos los casos por un marcado aumento, que puede atribuirse a causlas socioeconómicas, medio ambiente y diferencias en las determinaciones ya que las pruebas espectrofotométricas no son tan exactas como el análisis por cromatografía de que desafortunadamente no disponemos.

\section{RESUMEN}

Se estudia el líquido amniótico, obtenido por punción vía vaginal en 100 mujeres con embarazo a término, en trabajo de parto.

Se determina colesterol, fosfolípiidos, triglicéricos y lípidos totales.

\section{SUMMARY}

The amniotic liquild is studied. which is obtained by vaginal puncture on 100 pregnant on labor.

Cholesterol, phospholipids, trigliicerids and total lipids are determined.

\section{BIBLIOGRAFIA}

1․ - Evans J. Jerry. - Prediction of respiratory distress syndrome The new England journal of medicine. 292-21 pg. 113-115, 22 de marzo de 1975.

2․ - Cortés C. y Col. - Anticonceptivos orales, lipidos totales y colesterol. Tribuna Médica. 624 - LIII- № 8 pg. 43 - 1976.

3․ - Cortés C. Motta D. Caballero D. - Revista Colombiana de Obstetricia y Ginecología XVII - 5 - 297 - 300 Octubre 1976.

4?. Copeland B. - Control de calidad analítica 14 - 1977.

5: - Técnica para la determinación de Colesterol en el colorímetro Spekol manual de Instrucciones.

6․ - Fosfolípidos - Test Achebe. Instrucciones de empleo.

7ํ - Triglicéridos - Test Achebe. Instrucciones de empleo.

$8^{\circ}$ - Diagnostica Merck: Instrucciones de empleo. Merck Alemana.

9․ - Hytten Frank E. Lind Tom. - índice para el diagnóstico durante el embarazo. Pág. 104-106 - 1974. 